\title{
Posterior mediastinal neuroblastoma masked as flaccid paraparesis in a 3 year child
}

Farah S. Yahya, $M B C H B, C A B P$, Hieder A. Al-Shami, MD, EFNS, MRCS.

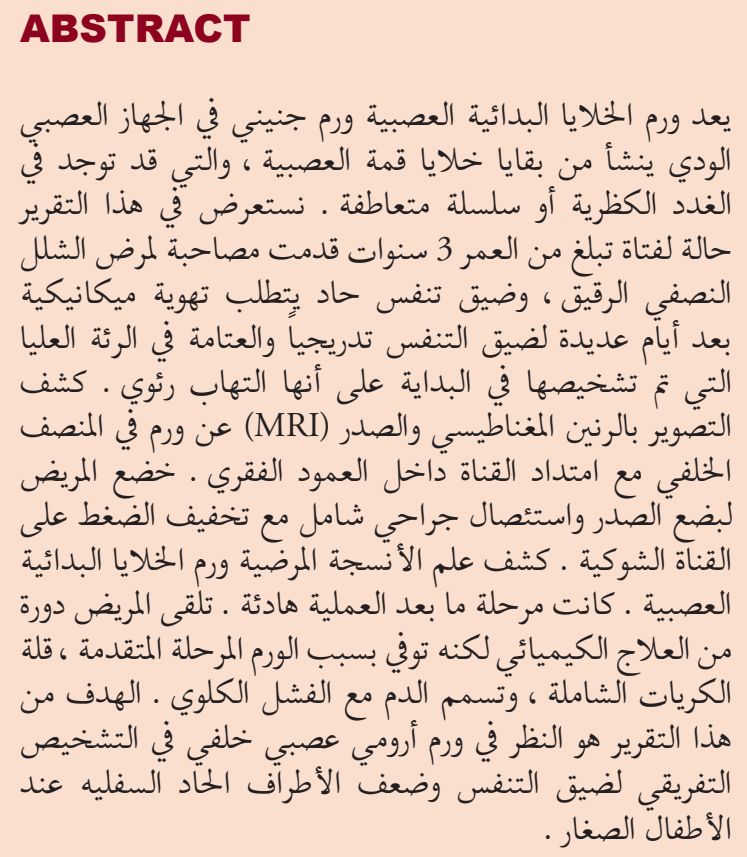

Neuroblastoma is an embryonic tumor of the sympathetic nervous system originating from neural crest cell remnants, which may be found in the adrenal glands or sympathetic chain. We report a case of a three-year-old girl who presented with flaccid paraparesis, and acute respiratory distress that required mechanical ventilation after a multiple-day history of gradual-onset dyspnea and right upperlung opacity that was initially misdiagnosed as pneumonia. Chest and spinal magnetic resonance imaging (MRI) revealed a tumor in the posterior mediastinum with intra-spinal canal extension. The patient underwent thoracotomy and surgical en bloc resection with spinal canal decompression. Histopathology revealed neuroblastoma. The postoperative period was uneventful. The patient was administered a course of chemotherapy but died due to advanced stage tumor, severe pancytopenia, and septicemia with renal failure. The aim of this report is to consider posterior mediastinal neuroblastoma in the differential diagnosis of dyspnea and acute lower limb weakness in young children.
Neurosciences 2019; Vol. 24 (4): 320-323 doi: 10.17712/nsj.2019.4.20190036

From the Department of Pediatrics (Yahya), College of Medicine, University of Mosul, Mosul, Iraq, and from Department of Neurosurgery (Al-Shami), Al-Ahly Bank Hospital, Cairo, Egypt.

Received 23th April 2019. Accepted 23th July 2019.

Address correspondence and reprint request to: Dr. Farah S. Yahya, Department of pediatrics, College of Medicine, University of Mosul, Mosul, Iraq. E-mail: farahuthman82@gmail.com

ORICID ID: https://orcid.org/0000-0002-4965-6073

A fter leukemias and brain tumors, neuroblastoma $\mathrm{A}_{\text {is the third most common cancer in infants and is }}$ the most common extracranial solid tumor in children. It is an embryonal tumor of the sympathetic nervous system. ${ }^{1}$ It originates from neuroblasts. Approximately two-thirds of cases present as an abdominal mass. Thoracic (posterior mediastinal) neuroblastomas are asymptomatic and may be diagnosed accidentally by imaging or may become symptomatic after invasion of the neural foramen. ${ }^{2}$ In this case report, we highlight the challenges in the early diagnosis of posterior mediastinal neuroblastoma as one of the causes of acute lower limb weakness in young children.

Case Report. Patient information. A 3-year-old female child with no significant past medical problems and unknown immunization history presented with a history of dry cough and gradual onset of dyspnea for 2 weeks without fever or evidence of upper respiratory tract infection. She began to develop significant weight loss and progressive fatigue. Chest X-ray showed a heterogeneous opacity involving most of her right lung

Disclosure. The authors declare no conflicting interests, support or funding from any drug company. 
(Figure $1 \mathrm{~A} \& \mathrm{~B})$. The initial diagnosis was pneumonia, for which she was she was kept on antibiotics (amoxicillin) and bronchodilators. However, this treatment failed to improve her cough or dyspnea. Several days later, the patient developed weakness of both lower limbs which then progressed to a complete inability to walk, with flaccid weakness of her lower limbs over 20 days in addition to dyspnea. She was admitted to a local hospital. The main differential diagnosis at that time was Guillain-Barré syndrome, depending on history and clinical findings, only because the facility for other sophisticated investigation was unfortunately unavailable at that peripheral hospital. Her condition rapidly worsened, including further worsening of her dyspnea and the development of central cyanosis. She was referred to our tertiary center for immediate mechanical ventilation.

Clinical findings. Upon arrival, the patient was severely dyspneic with central cyanosis, respiratory rate of 50 cycles/minute, and peripheral capillary oxygen saturation $\left(\mathrm{SpO}_{2}\right)$ of $85 \%$ on room air. Neurological examination showed loss of sphincter control such that the bladder was palpable up to the level just below the umbilicus; therefore, she underwent catheterization. Further examination showed 2 palpable small right-side supraclavicular lymph nodes. Chest auscultation showed poor air entry in most of her right lung. Neurological examination of her lower limbs revealed flaccid paraparesis with loss of tone and reflexes and equivocal Babinski reflex on both sides. Sensory examination was difficult and non-conclusive and opsoclonus-myoclonus

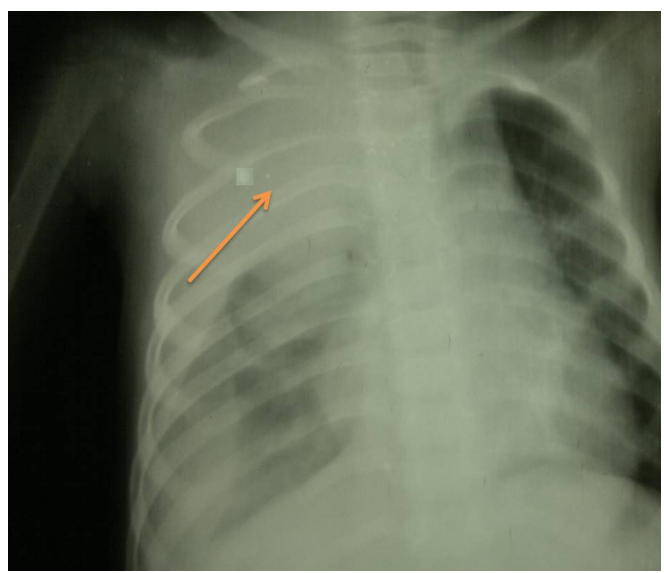

Figure 1 - Chest X-ray posterior-anterior view showing rather a well defined large homogenous soft tissue opacity lesion in the right upper zone area (arrow) that have broad based toward mediastinum and making an obtuse angle with it. There are no any detected calcifications seen within the lesion. The mass have overlapped the upper thoracic spine but not Silhouetting the upper cardiac border suggested to be retro-cardiac. Note the widening of the right sided upper posterior rib interspace highly localizing mass lesion to be in the posterior mediastinum.
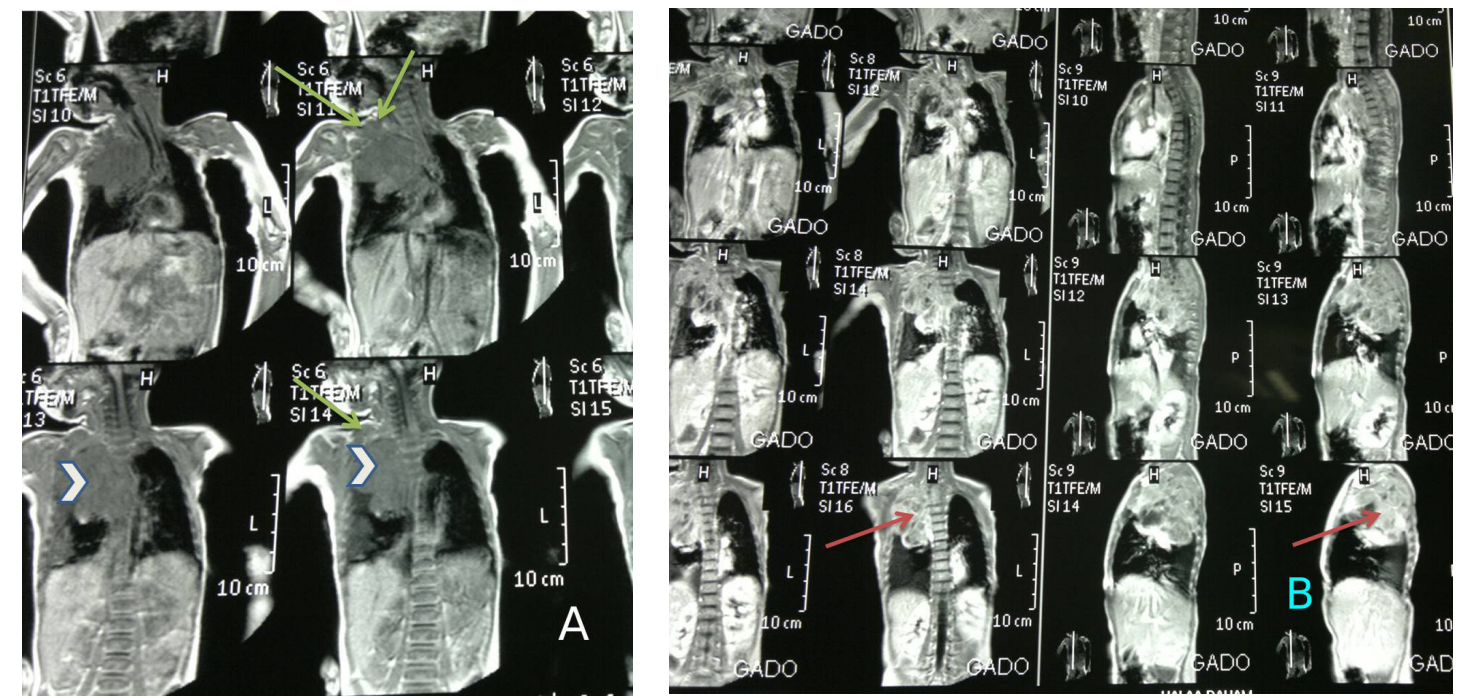

Figure 2 - Chest MRI (multiple sections through the chest and mediastinum). The examination showing A) large well defined soft tissue lesion about 8 * 6 $* 5 \mathrm{~cm}$ (arrow head), have low signal intensity in T1W image with multiple signal void area in favouring of few spots of calcification, This mass being extra pulmonary lesion at the right side para-vertebral area showing extension upward occupying the right side upper posterio-superior thoracic gutter connecting to another mass at the right side supraclavicular area in favour of multiple discrete $\&$ matted involved lymph nodes (arrow) by metastasis that have the same mass criteria in signal intensity enhancement. B) The whole 2 bulk masses showing heterogeneous enhancement post contrast (arrow). There are no any surrounded bony changes. This large mass abutting the descending thoracic aorta displacing trachea to the left side. 

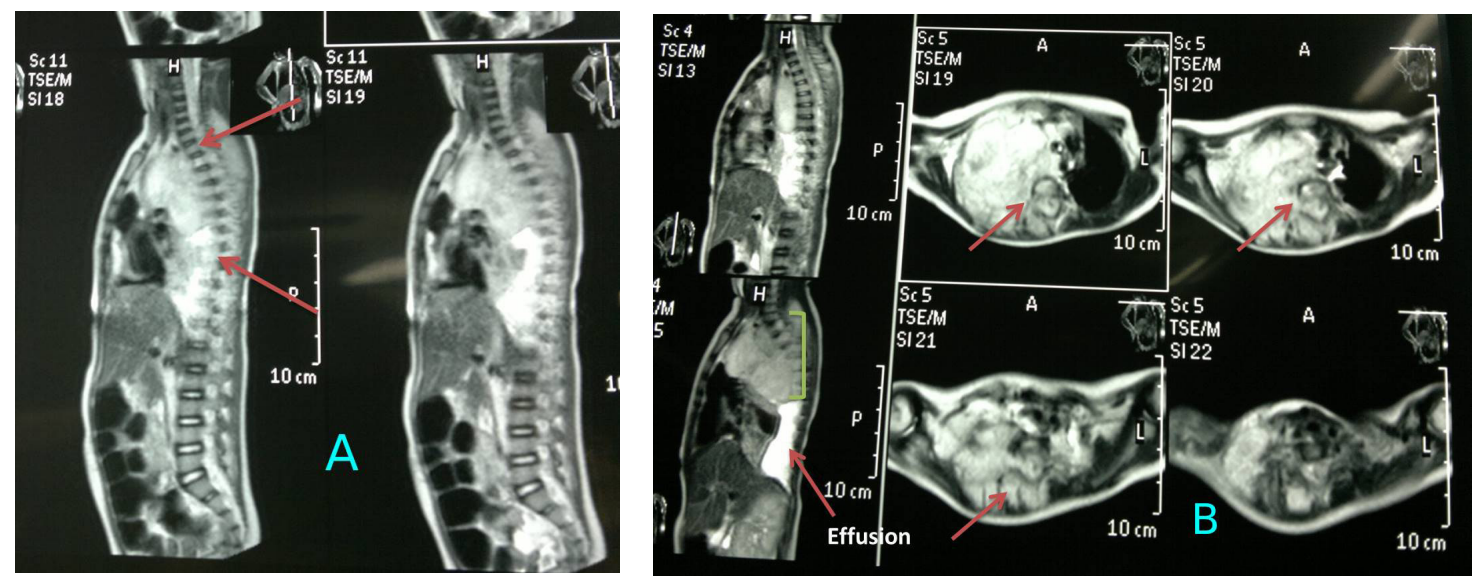

Figure 3 - Spinal MRI (multiple sections through the thoracic spine showing: A) large mass lesion $8{ }^{*} 6^{*} 5 \mathrm{~cm}$ enhanced heterogeneously in the right sided posterior mediastinum (arrows) that extended through the neural exit foramen in the right side aspect widened them at multiple levels D1-D8. The mass demonstrated also posterior extra dural component within the thoracic spinal sac deviating \& flattening the spinal cord aside from D1 to approximately D8 but not invade spinal cord tissue (arrows). B) Axial view the mass considered to be a ( dumbbell )malignant neoplasm (arrow), primarily in the mediastinum with posterior extension through an intervertebral foramen to the spinal canal \& causing spinal cord compression (extra dural extension) highly in favour of posterior mediastinal Neuroblastoma. There are a concomitant right sided pleural effusion that indicates involvement of the pleural cavity.

movement of both eyes were observed; however, her pupils were normal in size and reacted to light with no periorbital ecchymosis.

The patient was admitted to the respiratory care unit and ventilated with synchronized mechanical ventilation (SMV) for her declining oxygen saturation and worsening dyspnea.

Diagnostic assessment. The patient's serum electrolyte levels were normal and other blood test results were unremarkable except for respiratory acidosis. Emergency magnetic resonance imaging (MRI) of the chest showed a large right posterio-superior mediastinal mass that had pushed the trachea to the left with heterogeneous enhancement associated with pleural effusion, as well as 2 supraclavicular lymph nodes, direct invasion of the dorsal spine, and canal stenosis. The liver was normal no metastasis (Figure $2 \mathrm{~A} \& \mathrm{~B}$ ).

Whole-spine MRI showed extension of the tumor to the intraspinal canal at the level of D1-D8 (Figure 3A\&B). The initial differential diagnosis was neuroblastoma. Thereafter, assessment of urine vanillylmandelic acid (VMA) showed high levels. Bone marrow biopsy confirmed infiltration with neuroblastoma tumor cells.

Therapeutic intervention. Due to spinal compression, which is an oncologic emergency, the patient underwent thoracotomy and total resection of the mass with spinal decompression and relief of the canal stenosis with pedicle screw instrumentation to fix the spine after long-segment decompression.
Follow-up and outcome. Histopathological examination detected a poorly differentiated neuroblastoma as a small, blue, round-cells tumor with a fine chromatin pattern and high mitotic rate with nests of cells (pseudorosettes). Supraclavicular lymph node biopsy detected features of round blue cells consistent with neuroblastoma. According to the revised Shimada grading system, the tumor was a poorly differentiated neuroblastoma. Therefore, according to the International Neuroblastoma Risk Group Staging System (INRSS), the patient was classified as having high-risk neuroblastoma. ${ }^{2}$

The postoperative period passed smoothly with significant improvement of the dyspnea followed by weaning from the ventilator but with residual lower limb weakness, opsoclonus myoclonus eye movement, and loss of bladder control. Then, the patient was administered a course of induction of intensive chemotherapy including the combination of cyclophosphamide, etoposide, and vincristine; however, the patient unfortunately died during the induction phase due to severe pancytopenia, overwhelming septicemia, and renal impairment despite intensive care and management. The timeline of patient case history and follow-up is shown (Figure 4).

Discussion. Patient perspective. We reported a case of acute lower limb weakness in a young child which had masked an underlying mediastinal neuroblastoma. Acute 
flaccid paralysis is a pediatric emergency which includes many differential diagnoses such as poliomyelitis (which remained non-eradicated in the patient's locality), Guillain-Barré syndrome (GBS), trauma, transverse myelitis, and periodic hypokalemic paralysis. Every pediatrician should be aware of the common differential diagnoses of acute lower limb weakness in children, However, malignancy is an uncommon underlying cause of acute lower limb weakness in young children. Therefore, prompt evaluation of such cases is essential to exclude uncommon and malignant underlying causes. Our patient was initially misdiagnosed with acute flaccid paraparesis due to GBS due to the poor facilities in the hospital in which she was first managed before referral to our tertiary center. In this case report, we emphasize the importance of awareness of neuroblastoma as one of the potential underlying causes of spinal cord compression in young children. There are a few similar reported cases in the literatures. ${ }^{3}$ Between 7 and $15 \%$ of children with neuroblastoma present with spinal cord involvement. ${ }^{4}$ Chemotherapy and laminectomy are the modalities of choice for spinal compression caused by neuroblastoma. Spinal cord compression signs and symptoms including pain, weakness, sensory disturbances, and sphincter dysfunction are well known by every clinician. However, in young children, the detection of spinal cord compression is very difficult because of the subtle and gradual onset of the neurological symptoms; thus, it is difficult to identify the symptoms in young children, which leads to delayed diagnosis and serious neurological outcomes, as occurred in our patient. Young children refuse to walk and may even lose bowel and/or bladder control when they are ill. Thus, parents may miss early limb weakness. Therefore, the diagnosis is challenging for clinicians unfamiliar with normal motor milestones in children of such a young age. Emergency MRI is the imaging modality of choice to provide adequate clues in suspected cases.

Neurological recovery is correlated with early diagnosis of the initial symptoms. Caretaker or parent neglect and ignorance, unfortunately, leads to delayed diagnosis and advanced stage, resulting in dismal outcomes, as occurred in our patient.

Due to the bulk of the tumor with advanced stage, delayed diagnosis, and the use of combination chemotherapy, we unfortunately lost the patient during the induction phase due to refractory pancytopenia, overwhelming septicemia, and renal failure despite careful care and blood product support with broadspectrum antibiotics and early dialysis.

Conclusion. Malignant spinal cord tumors are a disastrous cause of acute lower limb weakness in children. Neuroblastoma is a common extracranial solid malignant pediatric tumor. Posterior mediastinal neuroblastomas comprise $15 \%$ of all cases of neuroblastoma as a primary site. The patients at high risk are those older than 18 months of age with disseminated tumors. Spinal cord compression symptoms and signs are subtle and diagnosis is especially challenging in young children. Mediastinal neuroblastoma can be asymptomatic and detected incidentally by imaging; it can also be symptomatic, causing respiratory or neurological symptoms if it grows through the spinal foramen into the spinal canal. Therefore, posterior mediastinal neuroblastoma should be considered in the differential diagnosis of dyspnea and acute paraparesis in young children.

Acknowledgements. We are gratefully acknowledge Dr. Hadeel M. Al-Hialy, Department of Radiology, College of Medicine, University of Mosul, Mosul, Iraq, for her kind review and comments on $X$-ray and MRI images. We would like to thank Editage (www.editage. com) for English language editing.

\section{References}

1. Lacayo NJ, Davis KL. Pediatric Neuroblastoma. [Internet]. [Cited 9 Oct 2017; Accessed 11 April 2019]. Available from: https:/emedicine.medscape.com/article/988284-overview

2. Shohet JM, Nuchtern JG. Clinical presentation, diagnosis, and staging evaluation of neuroblastoma [Internet]. [Cited 17 Feb 2018; Accessed April 2019]. Available from: https://www. uptodate.com/contents/clinical-presentation-diagnosis-andstaging-evaluation-of-neuroblastoma

3. Chauhan G, Bhatia, P, Bhardwaj A, Mittal S, Sharma P. Widely Disseminated Neuroblastoma Presenting as Acute Flaccid Paralysis in a Two Year Old Child: A Clinical Dilemma. International Journal of Contemporary Medicine 2014; 2: 161-163.

4. Shohet JM, Nuchtern JG, editors. Treatment and prognosis of neuroblastoma[Internet]. In: Shah S, editor. UpToDate. [Cited Feb 2018; Accessed 17 April 2019]. Available from: https:// www.uptodate.com/contents/treatment-and-prognosis-ofneuroblastoma 